\title{
Individualized ACL surgery
}

\author{
Jon Karlsson ${ }^{1} \cdot$ Michael T. Hirschmann $^{2} \cdot$ Roland Becker $^{3} \cdot$ Volker Musahl $^{4}$
}

Published online: 11 July 2015

(C) European Society of Sports Traumatology, Knee Surgery, Arthroscopy (ESSKA) 2015

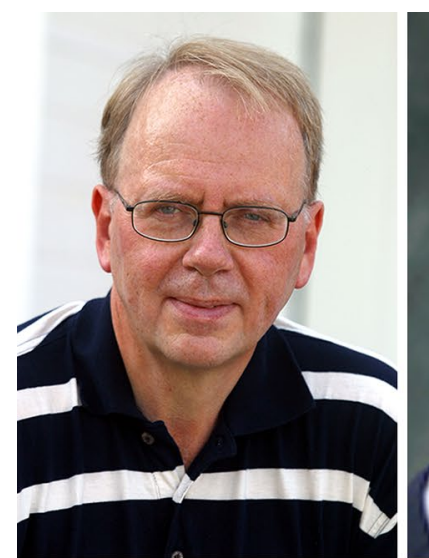

Jon Karlsson

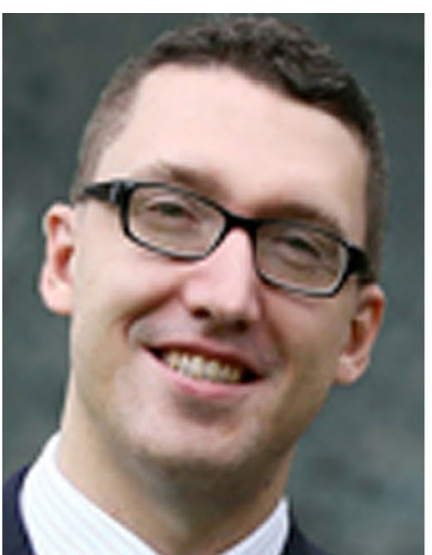

Michael T. Hirschmann

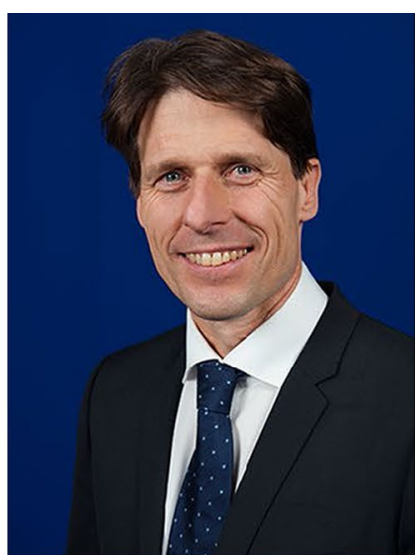

Roland Becker

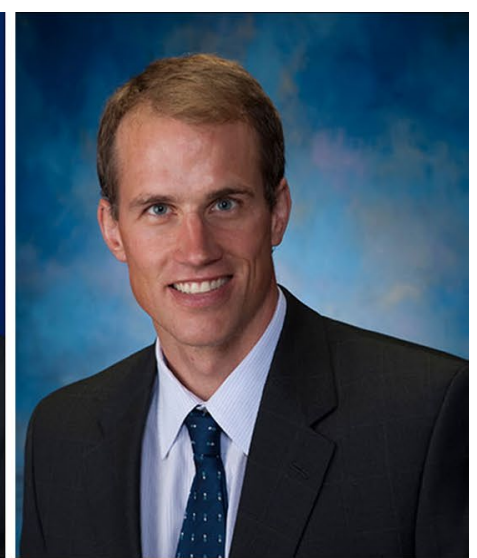

Volker Musahl
Jon Karlsson

jon.karlsson@telia.com

Michael T. Hirschmann

Michael.Hirschmann@unibas.ch

Roland Becker

roland_becker@yahoo.de

Volker Musahl

musahlv@upmc.edu

1 Department of Orthopaedics, Sahlgrenska University Hospital, 43180 Mölndal, Sweden

2 Department of Orthopaedic Surgery and Traumatology, Kantonsspital Baselland (Bruderholz, Liestal, Laufen), Bruderholz, Switzerland

3 Department of Orthopaedic and Traumatology, City Hospital Brandenburg, Hochstrasse 29, Havel, Brandenburg 14770, Germany

4 Department of Orthopaedic Surgery and Bioengineering, Orthopaedic Robotics Laboratory, UPMC Center for Sports Medicine, University of Pittsburgh, 3200 S Water Street, Pittsburgh, PA 15203, USA
The leading paper in this issue of the journal is entitled "Is the native ACL insertion site" completely restored "using an individualized approach to single-bundle ACL-R" [3]. This is rather a philosophical question than a statement. It is also applicable to many other aspects of ACL surgery, even knee surgery as a whole.

The burning questions more clearly are: How much can we really gain in individualized treatment procedures? How accurate does individualized treatment lead to restoration of native anatomy and improved function?

Aiming for more individualized ACL surgery, the individual patient needs and expectations as well as native anatomy have to be identified and characterized. Pre-operatively, a detailed history and clinical examination as well as improved imaging will help to better identify such individual characteristics of the ACL.

The core message of the leading article is that the surgeon has to intra-operatively understand and recognize the patients' individual anatomy and injury pattern. Not all ACL injuries are the same! 
The ACL insertion site anatomy varies in size and shape [2]. The relationship of the ACL to the lateral meniscus insertion on the tibia is variable. The ACL inserts in line with and medial to the anterior horn of the lateral meniscus, sometimes in isolation and sometimes curving around the anterior horn [5]. The insertion on the femur is constrained by the intercondylar and bifurcate ridges as well as the fellow ridge, which can vary considerably in curvature and its relation to the posterior aspect of the lateral femoral condyle [4]. Remnants of the native ACL can also vary in size and utility. The surgeon needs to decide whether to resect or at least partially incorporate ACL remnants into the reconstructed ACL construct.

The article by Middleton et al. challenges surgeons to use these pre- and intra-operative measuring tools to perform self-critique! They ask whether 70-80\% footprint fill is enough. But there is also a hidden question which is: Will the surgeon be able to restore even half of the native footprint when performing a transtibial ACL reconstruction with an 8-mm hamstring graft on a patient whose footprint was not assessed but in reality measures $20 \mathrm{~mm}$ ? The answer is probably not.

We all know that there have been major advances in the understanding of ACL reconstructive surgery during the last years. One of the main reasons for this is new understanding of the detailed anatomy of the knee. In a particular study, 45 patients were studied, all underwent singlebundle ACL reconstruction and the length and width of the native insertion sites were carefully measured. This emphasizes one thing, i.e. the importance of intra-articular measurements. In other words, we must with the accuracy of millimetres know exactly what we are doing inside the knee, where we place the drill holes and the exact location of the ACL graft. This paper also underlines that we need to be aware of the individual considerations. We place the emphasis on being "anatomic", instead of working on a specific technique. Single bundle or double bundle does not really matter, as long as we are "anatomic".

The so-called "individualized surgery" means that we tailor the entire treatment to each individual's knee, the size of the grafts, the tunnel angles, the graft tension and the native insertion sites. We cannot rely on the same technique for all knees. Sometimes, single-bundle ACL reconstruction could be the best option, sometimes double-bundle ACL reconstruction. But, we should also remember that many ACL-reconstructed knees using the transtibial technique also did well. Why is that? Today, we still have no clear answer to this question. The same research group from the University of Pittsburgh thought out to answer this question partly by assessing intra-graft forces in ACL grafts placed in different orientations. Robotic technology differentiated between anatomically placed grafts (high force) and non-anatomically transtibially placed grafts (low force) [1]. Grafts with low forces would be at a lesser risk of failure. These forces might propagate through the tibiofemoral joint contact in turn altering the risk of post-traumatic osteoarthritis.

But, the answer might be due to individual factors, i.e. some patients might not even need "anatomic" ACL reconstruction. And, also some patients adapt, lower their activity level and never return to sports activity. For that reason, some (or even many) patients are not really in need of surgical correction of the torn ACL. Studying individuals and large population cohorts alike will bring us further to answering the question of need for surgery.

The exact placement of the tunnels within the native footprint appears to be the most important factor in anatomic ACL reconstruction. Also complete coverage (at least "as complete as possible") of the ACL footprint has been considered important. The study by Middleton et al. [3] showed that the estimated insertion site coverage on the tibial site was approximately 70 and $80 \%$ on the femoral site. The importance of exact measurement is emphasized. This is still a pilot project, and we still do not know exactly how much of the native footprint needs to be covered. What we are currently doing is working with "the best available substitute", but how close to the original native anatomy we need to be can still be questioned.

\section{References}

1. Araujo P, Asai S, Pinto M, Protta T, Middleton KK, Linde-Rosen M, Irrgang JJ, Smolinski P, Fu F (2013) ACL graft position affects in situ graft force following ACL reconstruction. Paper presented at the International Society of Arthroscopy, Knee Surgery and Orthopaedic Sports Medicine. Toronto, Canada, 2013

2. Kopf S, Pombo MW, Szczodry M, Irrgang JJ, Fu FH (2011) Size variability of the human anterior cruciate ligament insertion sites. Am J Sports Med 39(1):108-113

3. Middleton KK, Muller B, Araujo PH, Fujimaki Y, Rabuck SJ, Irrgang JJ, Tashman S, Fu FH (2014) Is the native ACL insertion site "completely restored" using an individualized approach to single-bundle ACL-R? Knee Surg Sports Traumatol Arthrosc. doi:10.1007/s00167-014-3043-0

4. Sasaki N, Ishibashi Y, Tsuda E, Yamamoto Y, Maeda S, Mizukami H, Toh S, Yagihashi S, Tonosaki Y (2012) The femoral insertion of the anterior cruciate ligament: discrepancy between macroscopic and histological observations. Arthroscopy 28(8):1135-1146

5. Siebold R, Schuhmacher P, Fernandez F, Smigielski R, Fink C, Brehmer A, Kirsch J (2014) Flat midsubstance of the anterior cruciate ligament with tibial "C"'-shaped insertion site. Knee Surg Sports Traumatol Arthrosc. doi:10.1007/s00167-014-3058-6 